\title{
Combinatorial Chemistry in Ethnopharmacology
}

\section{John WM Yuen*}

School of Nursing, The Hong Kong Polytechnic University, Yuk Choi Road, Hung Hom, Kowloon, Hong Kong Special Administrative Region, Hong Kong

Biodiversity of natural products supplies an enormous source of drug discovery leads. It is the general belief that the nature of natural products are highly likely to provide therapeutic hits, because there are a greater number of stereocentres that intensifies scaffold diversity as supplied by the organic structures of more fused, bridged and spirocarbocyclic rings [1]. The successful isolation of resveratrol from grapes has remarked the benefits in promoting metabolic health by consuming red wines, suggesting the pharmaceutical values as a potential treatment for metabolic syndromes [2]. There are approximately $40 \%$ of all medicines especially, $60 \%$ of anticancer agents and $80 \%$ of antimicrobials available in clinical use are either natural products or their semisynthetic derivatives [3]. Synthetic forms of natural products have become the new trends of pharmaceutical development. Nowadays, classical drugs originally sourced from natural products become 'unnatural', such as anticancer Toxal (extracted from the Pacific yew tree) [4] and antimalarial Artemisinin (extracted from annual wornwood) [5], could be biosynthesized exploit multiple advanced techniques of genetic, enzymology and microbiology. However, such kind of success underlying the principle of 'make and test enough compounds and the right one will be found' often spend decades of time [6]. As reflected by many researchers, the challenge remains as the probability of finding the right one amongst the vast drug-like chemical space must be small, like looking for a needle in a haystack. Back to the middle of 1990s, Verdine [7] has already dictated the insights on the success of nature drugs discovery relies on building libraries in a combinatorial ways streamline with advances in techniques such as cell culture, compound extraction, high-throughput screening and synthesis. Combinatorial chemistry is now matured in drug development with the utilization of revolutionary computational simulation [8] and engineered biosynthesis [9].

In the era of 2000, the field of Ethnopharmacology has rapidly emerged. It studies the medicinal values of botanical substances, considering traditional herbal medicines (THM) as a major delivery. Historically, the use of herbal remedies has been served as a complementary but unique medical system around the world. Most herbal medicines are complex mixtures of largely unknown chemical composition. They may be decoctions, infusions, or extracts of one or many herbs. Given that the active ingredients of many herbs and their physiological actions are yet to be defined, the practice of THM is principally based on the experience accumulated for thousands of years. The body of science takes the initiative to turn the experiencebased THM into a scientific evidence-based context. This is an intricate task requiring huge amounts of manpower and efforts to screen, isolate, characterize and standardize the infinite number of chemical compounds and variants using empirical methods. From a single herb, the number of chemical structures extractable could be ranged from 10 -folds to hundreds. Taking a well-known medicinal mushroom called Ganoderma lucidum as example, over 300 molecules have been identified with more than 100 molecules isolated from each of the polysaccharides and triterpenes fractions as major bioactive ingredients [10]. The mushroom has demonstrated to possess various perceived health benefits including antidiabetic, antihypertensive, antioxidative, antimicrobial, immunomodulating, and antitumorigenicity [11]. These properties have widened the research spectrum of G. lucidum to investigate the disease-relevant mechanism in relation to the structural and physiological behavior of its raw extracts or individual isolated compounds, in addition to chemical profile and genome signature.
Vast amount of data generated from major research contributions must be recorded and assessable by building libraries of medicinal chemistry. It is the truth that medicinal chemistry space is expanding due to the continuous efforts in screening and testing many herbs and herbal formulations. Currently, several existing botanical databases have retained chemical structure and phytochemical data of traditional Chinese medicine, but many of these data are overlapping and in a non-standardized infrastructure format [12]. More importantly, none of these databases contains biological information that is significant for determining the bioactivity of chemical compounds. The main challenge here lies on how the accessible chemical space of medicinal chemistry can be related to the disease-relevant biological space, thus putting the way forward that the physiochemical properties are matching with certain chemical structures. This is aligned with the central thoughts of the mixture of diversity-oriented synthesis and biology-oriented synthesis - scaffold diversity of chemical synthesis must be increased in terms of functional groups and stereochemistry against the biological relevance to sanitize the medicinal chemistry databases into well-designed hierarchical 'scaffold tree' [1]. This is all about facilitating drug discovery and synthesis that Robb et al. [6] encouraged the collaboration in the drug discovery process, which relies on the sharing of ethnopharmacological knowledge, in order to be efficient the systematic design-makes-test should analyze emphasize the best compounds design before putting them into the test tubes. It is just a matter of time when combinatorial chemistry instrumentation becomes a commonplace for pharmaceutical manufactures that critical mass will be gathered through biotech-to-biotech collaborations. Natural products should remain as the major source to create molecule scaffolds or serve as building blocks in early-stage drug discovery, but more efforts are needed to boost pharmaceutical output $[13,14]$. To end this editorial, a collective inventory is desired for constructing medicinal chemistry library to catalogue information of natural products on structural features and variations, chemical functionalities, biological potency, inter-compound interactions, and possible biosynthesis pathways.

\section{References}

1. Barker A, Kettle JG, Nowak T, Pease JE (2012) Expanding medicinal chemistry space. Drug Discov Today 12: 1359-6446.

2. Tennen RI, Micgishita-kioi E, Chua KF (2012) Finding a target for resveratrol. Cell 148: 387-389.

3. Harvey A (2000) Strategies for discovering drugs from previously unexplored natural products. Drug Discov Today 5: 294-300.

4. Nicolaou KC, Yang Z, Liu JJ, Ueno H, Nantermet PG, et al. (1994) Total synthesis of taxol. Nature 367: 630-634.

*Corresponding author: Dr. John WM Yuen, School of Nursing, The Hong Kong Polytechnic University, Yuk Choi Road, Hung Hom, Kowloon, Hong Kong Special Administrative Region, Hong Kong, Tel: +(852)-2766-4130; Fax: +(852)-23649663; E-mail: john.yuen@polyu.edu.hk

Received December 15, 2012; Accepted December 17, 2012; Published December 19, 2012

Citation: Yuen JWM (2013) Combinatorial Chemistry in Ethnopharmacology. Mod Chem appl 1:e104. doi:10.4172/2329-6798.1000e104

Copyright: (c) 2013 Yuen JWM. This is an open-access article distributed under the terms of the Creative Commons Attribution License, which permits unrestricted use, distribution, and reproduction in any medium, provided the original author and source are credited. 
5. Mitchell W (2011) Natural products from synthetic biology. Curr Opin Chem Biol 15: $505-515$

6. Robb GR, McKerrecher D, Newcombe NJ, Waring MJ (2012) A chemistry wiki to facilitate and enhance compound design in drug discovery. Drug Discovery Today 12: 1359-6446.

7. Verdine GL (1996) The combinatorial chemistry of nature. Nature 384: 11-13.

8. Zhou X, Li Y, Chen X (2010) Computational identification of bioactive natural products by structure activity relationship. J Mol Graph Model 29: 38-45.

9. Ortholand JY, Ganesan A (2004) Natural products and combinatorial chemistry: back to future. Curr Opin Chem Biol 8: 271-280.
10. Yuen JW, Gohel MD (2005) Anti-cancer effects of Ganoderma lucidum: a review of scientific evidences. Nutr Cancer 53: 11-17.

11. Wachtel-Galor S, Yuen JW, Buswell JA, Benzie IFF (2010) Ganoderma lucidum (Lingzhi; Reishi): A medicinal mushroom. Taylor \& Francis CRC Press.

12. Barlow DJ, Buriani A, Ehrman T, Bosisio E, Eberini I, et al. (2012) In-silico studies in Chinese herbal medicines' research: evaluation of on-silico methodologies and phytochemical data source, and a review of research to date. J Ethnopharmacol 140: 526-534.

13. Persidis A (1998) Combinatorial Chemistry. Nat Biotechnol 16: 691-693.

14. Clardy J, Walsh C (2004) Lessons from natural molecules. Nature 432: 829837 